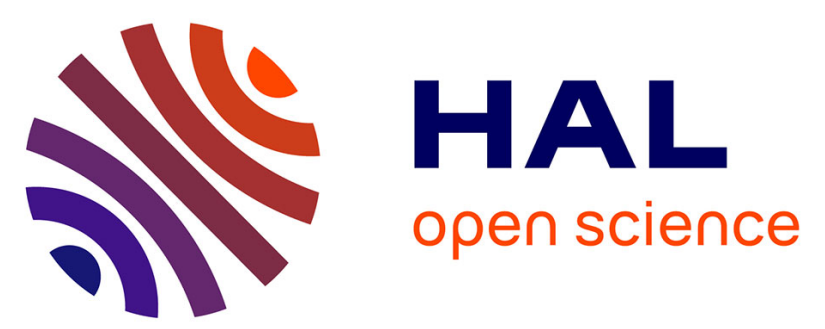

\title{
CHANGEMENT ORGANISATIONNEL ET IMPLEMENTATION DES TIC : POURQUOI FAIRE ATTENTION AUX DIMENSIONS ECONOMIQUES DE LA GESTION DU SAVOIR DANS LE CAS DES ERP?
}

Emmanuel Ruzé

\section{To cite this version:}

Emmanuel Ruzé. CHANGEMENT ORGANISATIONNEL ET IMPLEMENTATION DES TIC: POURQUOI FAIRE ATTENTION AUX DIMENSIONS ECONOMIQUES DE LA GESTION DU SAVOIR DANS LE CAS DES ERP ?. Vie et sciences économiques, 2011, 188, pp.38-56. hal-00655875

\section{HAL Id: hal-00655875 https://hal.science/hal-00655875}

Submitted on 3 Jan 2012

HAL is a multi-disciplinary open access archive for the deposit and dissemination of scientific research documents, whether they are published or not. The documents may come from teaching and research institutions in France or abroad, or from public or private research centers.
L'archive ouverte pluridisciplinaire HAL, est destinée au dépôt et à la diffusion de documents scientifiques de niveau recherche, publiés ou non, émanant des établissements d'enseignement et de recherche français ou étrangers, des laboratoires publics ou privés. 


\title{
CHANGEMENT ORGANISATIONNEL ET IMPLEMENTATION DES TIC : POURQUOI FAIRE ATTENTION AUX DIMENSIONS ECONOMIQUES DE LA GESTION DU SAVOIR DANS LE CAS DES ERP?
}

\author{
Emmanuel Ruzé
}

Dans cet article, nous proposons une réinterprétation d'études de cas traitant du couplage entre implémentation des ERP et changements organisationnels, et faisons appel pour cela à des outils de l'économie cognitive, jamais mentionnés auparavant dans l'étude des systèmes d'informations. Nous dégageons d'abord les spécificités de ce couplage et mettons en évidence l'importance cruciale de l'incertitude organisationnelle et de la gestion du savoir. Nous explicitons ensuite que les problématiques de coordination et de fiabilité complexes observées dans les stratégies des firmes peuvent non seulement être reproduites adéquatement par l'économie expérimentale mais aussi expliquées par un mécanisme économique simple et général.

We use cognitive economics tools, never mentioned before in Information systems studies, to provide an economic reinterpretation of case studies about coupling organizational change and ERP implementation. We set out its specificities and show the crucial significance of organizational uncertainty and knowledge management. We explain that economic coordination and complex reliability issues in firm strategies can be simulated by experimental economics and exhaustively explained by a simple and general economic model.

Economie cognitive, ERP, BPR, modèles mentaux partagés, usages des TIC. 
Cognitive Economics, ERP, BPR, shared mental models, ICT using.

Nous remercions entre autres Paul Bourgine, Frédéric Moatty, Hervé Dumez, François Eymard-Duvernay, Wilfrid Azan, Frédéric Deroïan, et plus généralement les chercheurs des Doctoriales 2004 du GDR TICS, de FORUM-GIFT, et du $4^{\mathrm{e}}$ colloque « La métamorphose des organisations » organisé par le GREFIGE de Nancy II pour leurs commentaires constructifs et leurs encouragements.

M290. 
Nous proposons une réinterprétation attendue d'études de cas traitant du couplage entre implémentation des ERP et changements organisationnels, et faisons appel pour cela à des outils de l'économie cognitive, jamais mentionnés auparavant en gestion. Nous dégageons d'abord les spécificités de ce couplage et mettons en évidence l'importance cruciale de l'incertitude organisationnelle et de la gestion du savoir. Nous explicitons ensuite que les problématiques de coordination et de fiabilité complexes observées dans les stratégies des firmes peuvent non seulement être reproduites adéquatement par l'économie expérimentale mais aussi expliquées par un mécanisme économique simple et général.

Nous explicitons pour cela certaines limites de la littérature existante: cette explicitation permet de caractériser une évolution du couplage entre deux objets de recherche en gestion des années 1990 ayant divisé les praticiens entre deux écoles de pensées (THOMAS, 2005). D’après BESSON (1999), durant la période 1994-1998, les directions d'entreprises, poussées entre autres par la mode, une littérature normative, et les cabinets de consultants, pensaient qu'implanter un ERP était une opportunité intéressante de restructurer au préalable ou en même temps l'organisation selon une logique de processes (Business Process Reengineering (BPR)). Seulement, on constate un écart croissant entre cette intention et les pratiques à partir de 1998 : les entreprises qui s'y essayèrent ont essuyé des échecs notoires. D'autres entreprises adoptent depuis une stratégie plus prudente d'implantation incrémentale au «coup par coup » (piecemeal) qui a davantage de succès, et qui suppose une succession des phases implémentation et de redesign des nouveaux processus de travail (ROBEY et al., 2002). Ces résultats synthétiques suggèrent la question suivante : comment expliquer cette évolution dynamique de la stratégie organisationnelle des entreprises?

Nous tentons plus précisément une explicitation des mécanismes économiques de «coopération cognitive» sur laquelle insiste LLERENA (1997), dont le thème central est celui des «modèles mentaux partagés». Elle implique, dans un cadre théorique réaliste relevant de 
la «rationalité limitée», une conception distribuée, située, de la cognition, et une attention particulière à la question de l'interprétation de l'environnement et du contexte.

La méthodologie inductive comporte deux aspects complémentaires :

- Un travail de «colligation» au sens de BLAUG (1994), i.e. une synthèse d'études de cas.

- Un rapprochement avec des travaux en économie expérimentale (BONINI et EGIDI, 1999) qui ont vocation à simuler des comportements organisationnels permet de reproduire de façon satisfaisante les faits observés. Enfin, ils peuvent être expliqués par un modèle économique plus général (HEINER, 1983).

\section{BREVE REVUE DE LITTERATURES}

Les ERP, sous-ensembles du système d'information assurant la gestion de plusieurs domaines de l'organisation et la collaboration des processus par des modules intégrés et un référentiel unique de données, sont des TIC complexes. Ils sont caractérisés par des «trajectoires sociotechniques » variées ; la littérature est prolifique, mais le phénomène ERP resterait un «mystère» (SEGRESTIN, 2003). Nous négligeons ici volontairement de nombreux travaux pour nous concentrer sur les limites de la littérature qui suivent, afin de suggérer qu'une approche économique cognitive des organisations concernées, associant les dimensions microanalytiques et dynamiques serait bienvenue.

Les ERP ont toujours entraîné des problèmes organisationnels sérieux (RAGOWSKY, 2002). En particulier, les bénéfices organisationnels d'une implémentation sont médiocres (SHANKS, 2000). Les aspects humains et organisationnels, fréquemment cités, restent mal compris, en particulier en ce qui touche les frictions provoquées par l'usage du progiciel (ASH et BURN, 2003). On sait peu de choses sur les conséquences de l'usage sur le management (GRANLUND, 2002). 
Les analyses des pratiques de Knowledge management et d'implémentation des ERP constituent, on le sait, deux littératures séparées. Les dimensions d'apprentissage, de partage du savoir n'ont pas été suffisamment analysées alors qu'elles sont d'importance (ASH et BURN, 2003). Cependant, la littérature en Management des Systèmes d'Information suggère que négliger les ERP est risqué lorsqu'il s'agit d'aborder les TIC où la gestion du savoir a un rôle de premier plan (BENDOLLY, 2003, Lee et Lee, 2000). Les aspects cognitifs commencent à être reconnus: AMOAKO-GYAMPAH et SALAM (2004) mettent en évidence de façon empirique le rôle des croyances partagées sur le succès de la technologie. SEGRESTIN (2003) fait même de l'étude de l'appropriation des ERP un «bon analyseur des modèles cognitifs» permettant l'innovation technique ou managériale, problématique négligée par les travaux traitant du «contrôle» et du «déterminisme technique». On constate également une prise de conscience de leur importance après implémentation : ainsi BIRONNEAU (2003) propose des étapes précises d'émergence des connaissances liées à l'usage du progiciel. Même la littérature qui évoque les succès d'implémentation en fonction des cultures nationales (VAN EVERDINGEN, 2003) souligne l'importance des variables cognitives «perception» et «rapport à l'incertitude», sur lesquelles nous reviendrons.

Peu d'études s'attachent à ouvrir la boîte noire de l'implémentation. ROBEY et al. (2002), divise la littérature anglo-saxonne en deux parties : le noyau dur de la recherche a considéré surtout les problématiques du terrain favorable à l'implémentation, des principales variables explicatives, des facteurs de succès (HONG, 2002), de l'estimation de la productivité (HITT, 2002). Une littérature plus marginale composée d'études de cas, a ouvert la boîte noire et s'est préoccupée de la question des procédures expliquant les succès, les changements observés.

Les ERP ont plutôt fait l'objet d'une attention des gestionnaires et sociologues (SEGRESTIN, 2003), mais une analyse mobilisant des outils issue de l'économie (hétérodoxe 
ici) n'a jamais été proposée, alors que le champ de recherche et les outils existent pourtant : la littérature sur l'économie de la connaissance, qui aborde la problématique des TIC et du changement organisationnel, n'en fait en effet pas mention. Or, l'étude de la dynamique d'implémentation où une lecture évolutionniste pourrait être bienvenue fait défaut.

\section{SOURCES ET SYNTHESE DES CAS.}

\section{Trois articles complémentaires abordent la thématique de la cognition dans les projets ERP}

Trois articles suffisent à dégager les données qualitatives qui nous intéressent ; de plus, notre proposition d'interprétation alternative peut s'appliquer à chacun d'eux. Cependant, nous insistons, nous sommes partis des limites intéressantes de Robey et al. (2002), qui fournit la majorité des données qualitatives.

C'est un travail comparatif de treize études de cas traitant d'entreprises très diversifiées, de taille homogène. Il propose d'une part une typologie des formes d'obstacles cognitifs (knowledge barriers) d'importance inégale lors de des phases d'implémentation des ERP, d'autre part une typologie des stratégies adoptées pour y faire face. Cette recherche a cependant des limites que ne cachent pas les auteurs: leur axe d'analyse théorique est l'opposition politique des intérêts et des valeurs, il permettrait d'expliquer les conséquences diverses des projets d'implémentation. Il est opposé à celui de l'apprentissage organisationnel, la «dialectique de la mémoire ancienne et des nouveau savoir». Or, l'article montre que leurs résultats inductifs ne correspondent pas clairement à la théorie mobilisée, comme le confesse honnêtement une note terminale. La description des processus est cependant fiable, ce qui nous autorise à nous appuyer sur elle. Involontairement donc, l'article insiste sur la question des difficultés d'implantation dues à des problèmes de gestion du savoir, d'apprentissage. 
Ce matériau était une opportunité intéressante : il était dommage de le laisser inexploité.

Nous l'avons complété par Besson (1999), une «autopsie cognitive des projets ERP», car ses travaux originaux proposent en effet la rupture chronologique qui nous intéresse en lien avec la thématique de Robey et al. (2002). Il a dégagé personnellement cette rupture à partir de vingt et une études de cas, tout en ayant le mérite de prendre du recul sur la mode qui consiste à implanter les ERP, et d'analyser les spécificités de ces logiciels. Bien que la thématique organisationnelle y soit centrale, il insiste en fait surtout sur la conception erronée que les concepteurs des ERP ont de l'organisation, ainsi que sur les dimensions conflictuelles de l'outil, et moins sur les aspects cognitifs de l'intégration, cependant largement présents.

Bironneau (2003), une autre synthèse de cinq études de cas représentatives confirme les résultats de Robey et al. (2002), mais offre aussi un complément essentiel pour comprendre les ERP. Il a en effet le mérite spécifique de traiter de la gestion des connaissances d'une façon explicite dans la problématisation : il détaille les points de tension ayant trait à l'usage des ERP, en particulier, «la codification, la saisie, le partage, et la mise à jour des informations, ainsi que la production de connaissance nouvelles», et met en évidence la nécessité d'une «stabilité» de l'organisation et de «l'homogénéité des structures cognitives des acteurs» lors de l'implémentation.

\section{Synthèse des études de cas ${ }^{1}$.}

Tout d'abord, la temporalité et les rythmes des deux stratégies diffèrent : la première stratégie (S-1) correspondrait à la période 1994-1998 (Besson, 1999). Elle est déclinante, mais toujours présente par la suite. La phase de BPR a lieu pendant l'intégration, ou même avant et pendant cette phase. L'approche est accélérée et les changements ont lieu de concert (concerted change process, Robey et al. (2002)). Dans la stratégie 2 (S-2) qui émergerait 
après 1998, la phase de BPR suit l'intégration. On remarque au pire une superposition brève BPR-intégration éventuelle. L'approche est incrémentale, au coup par coup (piecemeal change process).

C'est ici le point le plus important, l'incertitude organisationnelle est la variable explicative principale : elle augmente dans le cas de S-1, et diminue dans le cas de la S-2. Le BPR est en effet le facteur qui augmente l'incertitude : en effet, dans le cas de S-1, les agents doivent faire face à l'assimilation de ces nouveaux processus standardisés qui, entre autres, «coupent au travers les applications fonctionnelles» et supposent l'apprentissage de «nouvelles structures de management». D'un point de vue cognitif, les agents ont à surmonter non seulement les «barrières de configuration» (techniques) de l'ERP, mais également les «barrières d'assimilation». Or, l'un des principaux résultats de Robey et al. (2002) est que les obstacles cognitifs majeurs, les plus difficiles à surmonter pour les utilisateurs d'ERP ne sont pas les «barrières de configuration», mais les «barrières d'assimilation», c'est-à-dire l'assimilation de ces nouveaux processes de travail, dont la mise en place augmente l'incertitude organisationnelle. A l'inverse, dans le cas de S-2, les agents se trouvent dans un environnement plus stable: «la mise en place d'un ERP suppose une relative stabilité de l'organisation en terme de découpage de fonction, et d'interactions entre ces différentes composantes». Les agents n'ont à surmonter que les «barrières [cognitives] de configuration », les moins redoutables selon les auteurs.

En conséquence, l'environnement cognitif des agents dessine des modes d'apprentissages contrastés: dans $\mathrm{S}-1$, un excès d'incertitude du fait de la phase de BPR requiert une dose importante d'apprentissage, dont les échecs sont fréquents: les utilisateurs préfèrent «travailler autour» de l'ERP. L'entreprise tente pourtant d'optimiser un usage soi-disant déjà «approprié»... Dans S-2, on remarque à l'inverse une incertitude modérée due au seul

\footnotetext{
${ }^{1}$ Faute de place, nous avons dû faire le choix de résumer et ordonner les données essentielles, qui mériteraient
} 
apprentissage : les parties prenantes peuvent ici expérimenter et désapprendre, de façon progressive. Les succès d'apprentissage sont fréquents, mais des tensions surviennent suite aux nécessités techniques de l'implémentation.

Les contraintes sur l'organisation et le comportement des agents (dont les interdépendances réciproques sont décrites par Bironneau, 2003) suivantes sont créatrices d'une incertitude différenciée pour les deux stratégies: elles peuvent être surmontées par l'apprentissage, dans des conditions favorables toutefois:

1- L'apprentissage de nouvelles définitions des objets pertinents suite à l'intégration.

2- Le problème de la codification hétérogène des éléments à manipuler par les différentes composantes d'une organisation. La résolution de ce problème relève d'une problématique de la gestion de la connaissance, en particulier d'incitation à la codification.

3- Le problème de l'émergence d'un schéma mental partagé à propos de la configuration de l'organisation et la redéfinition des contours métiers, schéma mental qui est à coup sûr problématique en cas de BPR; son acquisition relève de la problématique de la gestion des compétences.

4- Les tensions dues à la saisie par chacun dans l'ERP des informations utiles à tous, l'évolution de l'information pertinente, et les mises à jour et reparamétrages. Ce point est fondamental, en lien avec l'émergence d'un schéma mental collectif. Ne pas saisir ces informations peut avoir des conséquences catastrophiques.

La prise en compte de l'organisation est à l'origine différente: S-1 est à dominante technique, l'image organisationnelle sous-jacente est la métaphore de la «machine » et de la «clôture ». La stratégie, centralisatrice, vise à réduire les asymétries d'information. La simultanéité intégration/PBR est un moyen de réduire l'autonomie des unités de 
l'organisation. L'intégration est globale, avec un très fort (et dangereux) couplage système/tâche et système/nouveaux processes. La totalité de l'organisation concernée par l'ERP est mobilisée lors du PBR, voire adaptée à l'ERP dans le pire des cas. S-2 est à dominante organisationnelle, l'image organisationnelle sous-jacente est celle de la communauté et de l'ouverture, et elle respecte les centres d'autonomie. Il n'y a pas de centralisation, et parfois même décentralisation. L'implémentation se fait sur quelques sites à la fois. D'où une réduction du couplage système/tâches, et le couplage système/nouveaux processes est différé. Les petites sous-unités (une fonction, voire l'équipe) sont mobilisées pour l'apprentissage et cela peut impliquer une réorganisation localisée des tâches. L'ERP est adapté à l'organisation.

Remarque: les conséquences en terme de coûts diffèrent, ce qui peut complexifier les arbitrages. S-1 est une tentative périlleuse de limitation des coûts en changeant tout en même temps. S-2 implique en revanche un risque de dérive des coûts. De plus, les gains à espérer de l'ERP sont différés.

\section{PROPOSITION D’EXPLICATION THEORIQUE}

- Nous mettrons en évidence expérimentalement les fondements microanalytiques des rigidités comportementales d'agents confrontés à un environnement organisationnel incertain, ces rigidités ralentissant ou annihilant l'apprentissage collectif, et ce, au moyen des concepts de «trappe cognitive» ${ }^{2}$ et de «coordination non-coopérative».

- Nous expliciterons au moyen du modèle de Heiner fondé sur le concept de «seuil de fiabilité » le mécanisme économique qui explique l’évolution observée.

\footnotetext{
${ }^{2}$ L'expression suggère que, durant une période d'ajustement à des conditions externes changeantes, les organisations sont prises dans des configurations («trappes») qui sont localement optimales seulement sur le court terme. Cette idée de trappe suggère l'idée de rigidité des comportements, en particulier que «les organisations ne seraient pas capable de «sauter » hors de la trappe et de se réorganiser elles-mêmes d'une façon plus efficiente (BONINI, 1999).
} 


\section{LES EXPERIMENTATIONS DE BONINI ET EGIDI (1999)}

Ce sont les seules à étudier le rapport entre le processus de routinisation en tant que phénomène organisationnel et la routinisation en tant que processus mental individuel, le deuxième étant la cause micronanalytique du premier. Cette question est traitée ici pour savoir si les «trappes organisationnelles» (organizational trap) sont fondées sur des «trappes cognitives individuelles» (individual cognitive trap) : plus concrètement, le phénomène empirique qui se manifeste lors des expériences est la rigidité des actions d'une « équipe » lors de sauts en complexité des interactions ${ }^{3}$. Cette question est traitée dans le contexte d'une expérience fondée sur un protocole utilisant le jeu de carte «Target The Two».

\section{Le jeu de Bonini et Egidi :}

L'article prend un certain recul théorique en commentant plusieurs expériences ayant pour cadre le jeu «Target The Two », qui a été conçu spécialement au laboratoire CEEL pour étudier la formation en laboratoire de comportements routiniers dans un contexte organisationnel décentralisé, en particulier celui du travail en équipe, qui suppose une interdépendance entre les actions et, a priori, une nécessité de coopération. Pour faire simple, dans ce jeu deux joueurs échangent des cartes masquées ou non afin d'aboutir à une configuration précise sur la table de jeu. Chacun des joueurs maîtrise une part d'incertitude en manipulant soit les couleurs, soit les chiffres de son jeu de cartes. Le jeu est a priori coopératif, car les gains sont partagés équitablement. Le jeu est répété, chaque coup est coûteux, les joueurs tentent de maximiser leurs gains collectifs et de minimiser le nombre total de coup à chaque fois. Les «cobayes», raisonnant en terme de sous-objectifs, découvrent par tâtonnements deux stratégies de trois «coups», à accomplir dans un ordre précis, permettant à coup sûr, si la distribution des cartes sur la table est simple, d'atteindre

\footnotetext{
${ }^{3}$ Le mot «trappe » est imprécis dans l'article sur un point pour nous crucial : pour une organisation il semble désigner une configuration; pour l'individu, il s'agit de la persistance d'un schéma mental. Nous en userons ainsi désormais.
} 
le but du jeu. Cela ne les empêche cependant pas d'en chercher d'autres (exploration) au besoin en configuration plus complexe.

Quelles lois déduire de ces expérimentations?

1/ L'existence en configuration simple de phénomènes de «dépendance par rapport au chemin »: le travail d'équipe est influencé par la façon dont les membres se coordonnèrent initialement entre eux («stratégie d'exploitation»).

2/ En configurations simples, contre l'intuition, les paires de joueurs ayant des stratégies de jeu flexibles ont de moins bons résultats que ceux ayant des comportements rigides ( cas précédent). Ces comportements rigides induisent moins de «signaux» ambigus, et donc réduisent l'incertitude entre les comportements.

3/ Au cas où les configurations de cartes sont plus complexes, deux cas sont possibles :

- Une coordination non-coopérative ${ }^{4}$ : elle suppose un modèle mental individuel plus simple, c'est la plus fréquemment observée, elle est sous-optimale mais d'une certaine efficacité. Ce type de coordination implique en revanche l'absence d'un «modèle mental partagé», par définition. L'apparition de ce type de coordination provoque l'apparition d'une «trappe organisationnelle» qui est la conséquence de la présence de «trappes cognitives» individuelles 5 .

- Coordination coopérative : elle demande un effort mental, qui consiste à se mettre à la place de l'équipier (Rôle, fonction, responsabilités...), et donc un modèle mental individuel

\footnotetext{
${ }^{4}$ Un comportement de «coordination coopérative », ou «collaborative », consiste, pour tout membre d'une équipe, non seulement d'être conscient des besoins et fonctions de ses collègues, mais également de travailler à créer les conditions favorables à la fois à ses actions et à celles de ses collègues (Bonini, 1999).

${ }^{5}$ Bonini démontre que le phénomène de path dependency n'est pas dû directement à l'interaction des joueurs, mais émerge d'une distorsion entre l'activité individuelle d'exploration mentale de chaque joueurs. C'est là que se trouve le lien important entre «modèle mental partagé» et «connaissance organisationnelle»: «this
} 
complexe. Elle est rarement observée dans les expériences, car sa réussite est incertaine : les joueurs à rationalité limitée risquent alors d'augmenter l'ambiguïté de leurs interactions. On n'observe pas de «trappe cognitive» individuelle. Elle serait optimale pour des agents fictifs à rationalité forte.

\section{Ces lois permettent de décrire et prédire les comportements observés}

Il s'agit d'abord des phénomènes habituels de dépendance par rapport au chemin, quelle que soit la stratégie choisie, qu'une phase d'apprentissage permet cependant de surmonter, dans des conditions favorables.

Il s'agit surtout de caractéristiques présentes dans S-1, c'est-à-dire celle où l'on remarque une forte incertitude organisationnelle; les caractéristiques problématiques et décisives en termes organisationnels sont les points 3/ et 4/ de la classification des contraintes techniques et organisationnelles énoncées ci-dessus. Ces dernières décrivent de façon satisfaisante les obstacles cognitifs que ROBEY et al. (2002) regroupent sous la dénomination «barrières d'assimilation» et «barrière de configuration» respectivement, $\mathrm{du}$ fait de l'importance de l'apprentissage requis au niveau organisationnel qui les caractérise.

D'une part (et c'est le point le plus décisif) le problème de l'émergence d'un schéma mental partagé concernant la configuration de l'organisation ( point 3/, connaissance ou non des processes de travail) correspond au problème principal mis en évidence dans l'article de Bonini, l'émergence problématique d'un «modèle mental partagé»: l'échec de cette émergence, de cette compréhension des conditions de travail des autres joueurs dans le cas du jeu TTT entraîne expérimentalement l'apparition de «trappes organisationnelles», situations où chaque joueur maîtrise ses actions, mais où le résultat collectif n'est pas optimal : «each member is principally concerned to control the conditions for his action and to perform his 
action correctly». L'augmentation de l'incertitude provoque une rigidification des comportements individuels et collectifs. Dans le cas des ERP, cela signifie que l'augmentation de l'incertitude (due au BPR) fige les processus de travail, et en particulier, ce qui est effectivement observé, ceux concernés plus directement par les applications du progiciel (assimilations knowledge barriers).

D'autre part, le problème des tensions cruciales liées à la saisie d'informations utiles à tous (point 4/), correspond à une autre caractéristique de la «coordination non-coopérative» de Bonini : la coordination coopérative, en effet, consiste justement, au travers des actions des joueurs, à fournir les informations permettant le travail d'autrui. Dans le cas des ERP, c'est justement ce manque de transmission correcte et coopérative des informations, qui provoque des échecs cuisants, en particulier une sous- ou non-utilisation du progiciel.

Si l'incertitude organisationnelle en 3/ est modérée, cette coordination coopérative peut cependant émerger, situation qui correspond à S-1.

Ces expérimentations ont donc clairement permis de mieux comprendre les mécanismes microanalytiques observés, car y faire varier l'incertitude permet de faire des prédictions de comportements homogènes à celles observées dans nos études de cas. Cependant le raisonnement économique sous-jacent manque encore: pourquoi la coordination noncoopérative et «l'absence de modèle mental partagé» sont-ils sélectionnés expérimentalement lorsque l'incertitude devient plus importante, bien que ces réactions soient sous-optimales?

\section{Le modèle de HEINER (1983)...}

Ce modèle montre que lorsque l'incertitude devient plus forte, les agents ont plus de mal à reconnaître les bonnes actions à inclure dans leur répertoire : le seuil de fiabilité de ces

particulièrement «knowledge relative to the functions, roles, and responsabilities to one's team mates». 
nouvelles actions diminue, et leur inclusion est plus restrictive. Le seuil de déclenchement de l'apprentissage des nouvelles actions est donc plus difficile à atteindre.

Le choix du modèle se justifie clairement: il est fondé d'emblée sur le niveau de connaissance de l'agent économique, l'écart entre ses compétences $\mathrm{C}$ et la difficulté $\mathrm{D}$ du problème de décision à résoudre (inexistant par exemple dans la théorie néo-classique). Cet écart est à l'origine de l'incertitude à laquelle l'agent doit faire face, incertitude qui provient de la complexité de l'environnement et des capacités limitées des agents. De plus, il se distingue d'autres modèles, car il décrit une structure de décision individuelle qui prête aux agents une rationalité systémique et procédurale (Laville, 1998), conforme aux comportements observés dans les organisations. Enfin, il est simple et parcimonieux (dans la version de 1983) et a une portée explicative très générale (Voir annexe pour la partie formalisée.).

\section{... ET SA PORTEE EXPLICATIVE}

Les implications de ce modèle d'exploration/exploitation correspondent à ce qui est observé dans le cas de l'implémentation des ERP en situation de complexité croissante. Elles concernent surtout un aspect original de la rationalité des agents qui avait manqué en fin de compte dans l'expérience de Bonini : le modèle de Heiner explicite clairement que, du point de vue de la décision individuelle, l'adoption de nouvelles actions n'est pas toujours rationnelle. En situation de complexité croissante, l'interprétation de l'environnement est incertaine, et la fiabilité du choix d'une nouvelle action également; l'augmentation du répertoire d'une nouvelle action conduirait à une augmentation des possibilités d'erreur de décision. Ces actions non sélectionnées sont, dans le cas de l'implémentation des ERP :

- Le non-apprentissage des nouveaux processes liés aux ERP, c'est-à-dire le point d'achoppement le plus décisif de l'intégration des ERP, qualifié d'«assimilation knowledge 
barriers» par Robey. Elles se caractérisent en particulier par les obstacles techniques et organisationnels majeurs 3/. Ainsi, le raisonnement sous-jacent au modèle de Heiner est une réponse, une proposition d'explication de la rationalité de cette absence pourtant sousoptimale d'un point de vue collectif de «modèle mental partagé» et de la présence de «coordination non-coopérative» observée.

- Le non-apprentissage des aspects techniques des ERP (Point 4/), qui correspondent aux «barrières de configurations», les moins importantes. Une augmentation de l'incertitude rend cette action elle-même incertaine.

Le modèle de Heiner seul suffit à expliquer les comportements observés par une modélisation analytique. Cependant, il ne permet pas de les reproduire, d'où le mérite complémentaire des expérimentations précédentes.

Remarque: d'autres modèles simples et classiques problématisent la coordination en économie, comme ceux fondés sur le dilemme du prisonnier en théorie des jeux (évolutionniste ou non). Cependant, la limite rédhibitoire de ces approches «mécaniques» est de ne pas prendre en compte de façon réaliste les dimensions cognitives du comportement des agents et l'incertitude dans l'environnement, alors qu'elles sont cruciales d'après nos études de cas.

\section{DISCUSSION ET CONCLUSION}

Dans un contexte de complexité croissante dû à des changements organisationnels des questions cruciales de gestion du savoir se posent dans une organisation qui implémente un TIC complexe, dont l'ERP est l'archétype. Notre apport est double :

- empirique : la confrontation des théories est essentielle dans tout recueil d'études de cas. Nous avons expliqué autrement les modes de coordination et les routines observées, éléments qui déterminent la sélection d'actions et stratégies fiables, conditionnent l'efficacité des 
stratégies observées et le succès d'une technologie. Notre travail est l'explicitation d'une proposition satisfaisante d'alternative théorique attendue par ROBEY et al. (2002), remis par ailleurs en perspective historique.

- théorique: les tentatives de tester l'articulation entre modèles mentaux et situations de coordination concrètes sont inexistantes (BONINI, 1999, que confirment FELIN\&FOSS, 2004). Notre travail permet aussi de nuancer la littérature évolutionniste qui affirme que les discordances des interprétations des individus d'un même groupe dans un environnement perturbé seraient la source de l'apprentissage organisationnel et une source d'accroissement des performances (COHENDET, 1998).

Certes, nous n'avons pu expliciter qu'un aspect empirique très succinct avec des limites méthodologiques évidentes, principalement dues à la nature secondaire des matériaux. Il serait intéressant de corroborer des hypothèses testables déduites facilement de notre cadre théorique sur d'autres études de cas, car il a un pouvoir prédictif. Il serait aussi fructueux de mettre en concurrence notre proposition d'explication avec celle d'ordre technique et historique qui lierait cette évolution des stratégies à l'externalisation des ERP sur des serveurs Internet (AZAN, 2003). 


\section{Le modèle de Heiner :}

L'écart C-D est conçu comme un vecteur : $U=u(p, e)$

« $\mathrm{P} »$ est une variable de perception qui correspond à la capacité de l'agent à déchiffrer les relations entre son comportement et l'environnement. Plus cette variable baisse, plus l'incertitude augmente. «E» est la synthèse des variables décrivant l'environnement, en particulier la complexité du problème de décision devant être résolu par l'agent. «U» est une incertitude de l'usage de l'information, c'est-à-dire une incertitude due à la difficulté d'un agent à capacités limitées à interpréter ${ }^{6}$ un environnement complexe. Pour quelle raison un agent possédant un répertoire fixe d'actions rajouterait-il dans ce répertoire une nouvelle action pouvant augmenter sa performance ? Soient :

- $\pi(\mathrm{e})$ : la probabilité que ce soit le bon moment de choisir cette action.

- 1- $\pi(\mathrm{e})$ : la probabilité que ce soit le mauvais.

Ces probabilités se rapportent aux situations, décrivent un état de fait probable, un lien entre comportement et situation. Heiner définit ensuite des «probabilités comportementales conditionnelles », qui se rapportent au niveau de l'agent seul. Deux situations sont alors possibles, à niveau d'incertitude donné : soit les agents choisissent la nouvelle action lors des bonnes circonstances, soit ils choisissent la nouvelle action lors des mauvaises. La structure de l'incertitude conditionne ces probabilités ${ }^{7}$ :

\footnotetext{
${ }^{6}$ Le modèle permet de construire un «lien» intéressant entre incertitude et complexité : «L'incertitude existe parce que les agents ne peuvent déchiffrer tout la complexité des problèmes de décision auxquels ils font face [...] la flexibilité du comportement qui consiste à réagir aux informations est contraint à en rester à des plus petits répertoires de comportements qui peuvent être administrés de façon fiable»

${ }^{7}$ Ces probabilités peuvent s'interpréter selon Heiner comme des erreurs de type 1 et 2 en théorie des tests. La distinction entre les deux types de probabilités est par ailleurs subtile : la probabilité $\mathrm{r}(\mathrm{U})$ est la probabilité que l'agent interprète de façon correcte une situation favorable donnée, apparue avec la probabilité $\pi(\mathrm{e})$ auparavant.
} 
- $\mathrm{r}(\mathrm{U})=\mathrm{r}(\mathrm{U}(\mathrm{p}, \mathrm{e}))$ la probabilité comportementale correspondant à la première situation («r» pour «right»).

- $\quad \mathrm{w}(\mathrm{U})=\mathrm{w}(\mathrm{U}(\mathrm{p}, \mathrm{e}))$ la probabilité comportementale correspondant à la deuxième $(\ll \mathrm{W} »$ pour «wrong»).

Chacune des deux situations apporte des gains ou pertes, qui sont relatives à l'environnement «e» :

- g(e) est le «gain» dû à l'inclusion de la nouvelle action dans le répertoire.

- 1(e) est la perte due à l'inclusion erronée de la nouvelle action («l» pour «loss»).

Puis, Heiner définit un ratio de fiabilité, qui compare les probabilités comportementales, la «chance» de sélectionner l'action lors des bonnes circonstances et lors des mauvaises : $\mathrm{r} / \mathrm{w}=\mathrm{r}(\mathrm{U}) / \mathrm{w}(\mathrm{U})$.

La condition de sélection d'une nouvelle action suffisamment fiable se formule ainsi :

$$
\begin{aligned}
& g(e) \times r(U) \times \pi(e)>1(e) \times w(U) \times(1-\pi(e)) \\
\Leftrightarrow & r(U) / w(U)>1(e) / g(e) \times(1-\pi(e)) / \pi(e) \\
\Leftrightarrow & r / w>T(e)
\end{aligned}
$$

Le premier membre est le ratio de fiabilité précédemment défini, et qui correspond à la fiabilité de la sélection pour l'agent de l'action en question. Le deuxième membre est une limite de tolérance, notée $\mathrm{T}(\mathrm{e})$, qui est seulement fonction de e, incertitude de l'environnement. 


\section{BIBLIOGRAPHIE}

AMOAKO-GYAMPAH K. et SALAM A.F. (2004), "An extension of the technology acceptance model in an ERP implementation environment", Information\&Management, vol. $41, \mathrm{n}^{\circ} 6, \mathrm{p} .731-745$.

ASH C.-G. et BURN J.-M. (2003), “A strategic framework for the management of ERP enabled e-business change”, European journal of operational research, vol. 146, n 2, p. 374387.

AUTEUR (2003), «Changements organisationnels et usages des TIC: quels rôles de la gestion du savoir? » Mémoire de DEA d'Economie, EHESS, septembre.

AZAN W. et TOURNANT L. (2003), Réussir votre projet ERP, Eyrolles.

BENDOLLY E. (2003), “Theory and support for processes frameworks of knowledge discovery and data mining from ERP systems", Information\&Management, vol. 40, $\mathrm{n}^{\circ}$ 7, $\mathrm{p}$. 639-647.

BESSON P. (1999), «Les ERP à l'épreuve de l'organisation », Systèmes d'information et Management, vol. 4, $\mathrm{n}^{\circ}$ 4, p. 21-51.

BIRONNEAU L. (2002), «Modélisation d'entreprise et pratiques de management implicitement liées aux ERP : enjeux conceptuels et études de cas », Finance Contrôle Stratégie, vol. 4, n 4, p. 29-50.

BLAUG M. (1994), La méthodologie économique, Paris, Economica.

BONINI N. et EGIDI M. (1999), « Cognitive traps in individual and organizational behaviour : some empirical evidence », Revue d'économie industrielle, ${ }^{\circ}$ 88, p. 153-187.

COHENDET P. (1998), «Information, connaissance, et théorie évolutionniste de la firme », L'économie de l'information, les enseignements des théories économiques, eds Petit P., Paris, La Découverte. 
FOREST G. (1999) «Généalogie des ERP et gestion des flux physiques », Systèmes d'information et Management, vol. 4, $\mathrm{n}^{\circ}$ 4, p. 71-89.

FRANCALANCI C. (2001), "Predicting the implementation effort of ERP projects : empirical evidence on SAP/R3", Journal of Information Technology, vol. 16, n 1, p. 33-48.

GEFEIN D. (2002) "Implementation team responsiveness and user evaluation of customer relationship Management : A quasi-Experimental Design study of Social exchange Theory", Journal of Management Information Systems, vol. 19, n 1, p. 47-69.

GRANLUND M. et MALMI T. (2002), "Moderate impact of ERP on management accounting : a lag or permanent outcome?" Management Accounting Research, vol. 13, $\mathrm{n}^{\circ} 1$, p. 299-321.

FELIN Teppo et FOSS Nicolaï J. (2004), “Organizational Routines, a Sceptical Look”, Becker Markus (eds), Handbook of Organizational Routines, Edward Elgar.

HEINER R. (1983), “The origin of predictable behavior”, American Economic Review, vol 4, $\mathrm{n}^{\circ} 4$, p. $560-595$.

HITT L.-M. et Wu D.-J., (2002) “Investment in enterprise resource planning : business impact and productivity measures", Journal of Management Information Systems, vol. 19, $\mathrm{n}^{\circ}$ 1, $\mathrm{p}$. $71-98$.

HONG K.-K., 2002, “The critical success factors for ERP implementation : an organizational fit perspective", Information \& Management, vol. 40, $\mathrm{n}^{\circ}$ 1, p. 25-40.

LAVILLE F., 1998, « Modélisation de la rationalité limitée : de quels outils dispose-t-on ? », Revue Economique, vol. 49, n² 2 , p. 335-365.

LEE Z. et LEE J. 2000, “An ERP implementation case study from an knowledge transfer perspective", Journal of information technology, vol. 15, n 4, p. 281-288. 
LLERENA D. (1997), «Coopérations cognitives et modèles mentaux collectifs, outils de création et de diffusion de connaissances », Economie de la connaissance et organisations, Entreprises, territoires, réseaux, eds GUILHON B. et alii, Paris, L'Harmattan.

MARKUS L. (2000), “Learning from adopters' experiences with ERP : problems encountered and success achieved", Journal of information technology, vol. 15, n 4, p. 245-265.

PARR A., 2000, "A model of ERP project implementation, Journal of information technology, vol. $15, \mathrm{n}^{\circ} 4$, p. 289-303.

RAGOWSKY A. (2002), "Special section : Enteprise Resource Planning", Journal of Management Information Systems, vol. 19, n 1, p. 11-15.

ROBEY D. et al. (2002), "Learning to implement Enterprise Systems : an exploratory Study of the Dialectics of Change", Journal of Management of Information Systems, vol. 19, ${ }^{\circ}$ 1, p. $19-46$.

SEGRESTIN D., «Les ERP, entre le retour à l'ordre et l'invention du possible », Sciences de la Société, p. 61.

SHANKS G. et SEDDON P. 2000, "Editorial”, Journal of Information Technology, vol. 15, $\mathrm{n}^{\circ} 4$, p. $243-244$.

STENSRUD E. (2003), “Alternative approaches to effort prediction of ERP projects", Information and software technology, vol. 43, $\mathrm{n}^{\circ} 7, \mathrm{p} .413-423$.

SUMMER M. (2000) "Risk factors in enterprise-wide/ERP projects", Journal of information technology, vol. $15, \mathrm{n}^{\circ} 4$, p. 317-327.

THOMAS J-L., ERP et PGI, sélection, déploiement, et utilisation opérationnelle, Dunod, 2005. 
VAN EVERDINGEN Y.-M. et WAARTS E. (2003), A multi-country study of the adoption of ERP systems : The effects of national culture, ERIM Working paper, Rotterdam School of Management. 